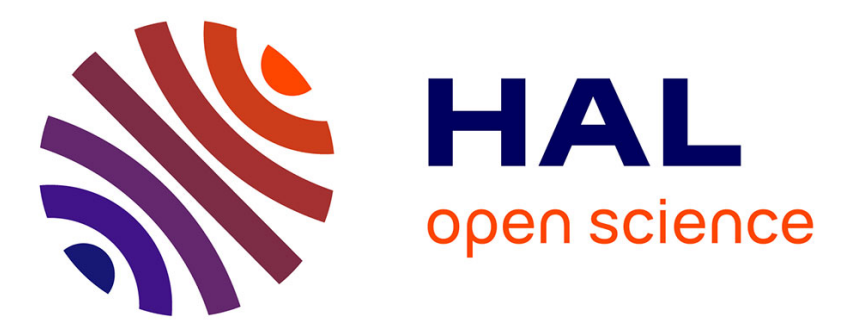

\title{
DISCUSSION OF THE CONVERGENCE PROPERTIES OF THE PERTURBATION SERIES USED IN THE CALCULATION OF EBIC- AND CL-CONTRASTS
}

W. Hergert, L. Pasemann, S. Hildebrandt

\section{To cite this version:}

W. Hergert, L. Pasemann, S. Hildebrandt. DISCUSSION OF THE CONVERGENCE PROPERTIES OF THE PERTURBATION SERIES USED IN THE CALCULATION OF EBICAND CL-CONTRASTS. Journal de Physique IV Proceedings, 1991, 01 (C6), pp.C6-45-C6-50. 10.1051/jp4:1991608 . jpa-00250693

\section{HAL Id: jpa-00250693 https://hal.science/jpa-00250693}

Submitted on 1 Jan 1991

HAL is a multi-disciplinary open access archive for the deposit and dissemination of scientific research documents, whether they are published or not. The documents may come from teaching and research institutions in France or abroad, or from public or private research centers.
L'archive ouverte pluridisciplinaire $\mathbf{H A L}$, est destinée au dépôt et à la diffusion de documents scientifiques de niveau recherche, publiés ou non, émanant des établissements d'enseignement et de recherche français ou étrangers, des laboratoires publics ou privés. 


\title{
DISCUSSION OF THE CONVERGENCE PROPERTIES OF THE PERTURBATION SERIES USED IN THE CALCULATION OF EBIC-AND CL-CONTRASTS
}

\author{
W. HERGERT, L. PASEMANN* and S. HILDEBRANDT \\ Martin-Luther-Universität, Halle-Wittenberg, Fachbereich Physik, Friedemann-Bach-Platz \\ 6, D-4020 Halle, Germany \\ *Asteilung Halbleiterphysik, Obever Eselsberg, Postflach 4066, D-7900 ULM, Germany
}

\begin{abstract}
For the calculation of the contrast of a defect in semiconducting material, the defect is regarded as a bounded region where the minority carrier lifetime $\tau$ ' is lower than that $(\tau)$ outside the defect region. The minority carrier density in the presence of the defect is normally written in terms of a perturbation series. The conditions for using a perturbation series for different kinds of defects are discussed.
\end{abstract}

\section{Introduction}

It has been shown that, by means of investigations of the electron beam induced current (EBIC) and the cathodoluminescence (CL), useful information can be obtained about the recombination activity, the depth $z_{D}$, or other geometrical parameters of crystal defects in the semiconductor sample. The precision and reliability of these parameters, obtained from the contrast, however, depend on the quality of the theoretical model used for the evaluation of the signal. One main point which influences the quality of the theoretical model is the description of the generation in contrast calculations. Recently it was shown [1] that the evaluation of the contrast profile area instead of the contrast profile itself enables the inclusion of realistic generation rates in the contrast calculations.

Another problem connected with the contrast calculations is the evaluation of the minority carrier density near the defect. Only in special cases it is possible to solve the problem exactly. Normally this calculation is performed using a perturbation expansion. Most of the calculations $[1,2,3$, $4,5,6]$ stop after the first order contribution, to get simple expressions which can be evaluated without great numerical effort. Pasemann $[7,8]$ has evaluated an approximate summation of the perturbation series for a surface parallel dislocation. In this work also the convergence of this perturbation expansion was discussed. In those papers, where the contrast calculations are restricted to the first order contributions, no great attention was dedicated to the fact, that it is not possible to use a perturbation series in each situation, characterized by the defect configuration and the recombination activity of the defect.

Due to this fact it is necessary to investigate the domain of convergence of the perturbation series in dependence on the parameters which enter the contrast calculation. Therefore, the aim of the paper is

- to formulate the criterions of convergence for some often studied defect configurations, 
- to discuss the quality of a first order approximation corresponding to the criterion of convergence.

A way to avoid the problems of convergence is to solve the threedimensional partial differential equation for the excess minority carriers numerically. This already was done ( cf.[9] ), but this way is connected with great numerical effort.

\section{Calculation of the contrast of defects}

The local recombination properties in a semiconductor sample are characterized by the radiative and non-radiative lifetimes of the minority carriers. $\tau_{r}(\vec{r})$ and $\tau_{n r}(\vec{r})$, respectively, which together form the total minority carrier lifetime $\tau(\vec{r})=\left(\tau_{r}^{-1}(\vec{r})+\tau_{n r}^{-1}(\vec{r})\right)^{-1}$. In the mostly used volume recombination model the defect is regarded as a bounded region of space characterized by a minority carrier lifetime $\tau^{\prime}$ which is lower than the lifetime $\tau$ of the the defect-free matrix region (cf. Fig. 1).

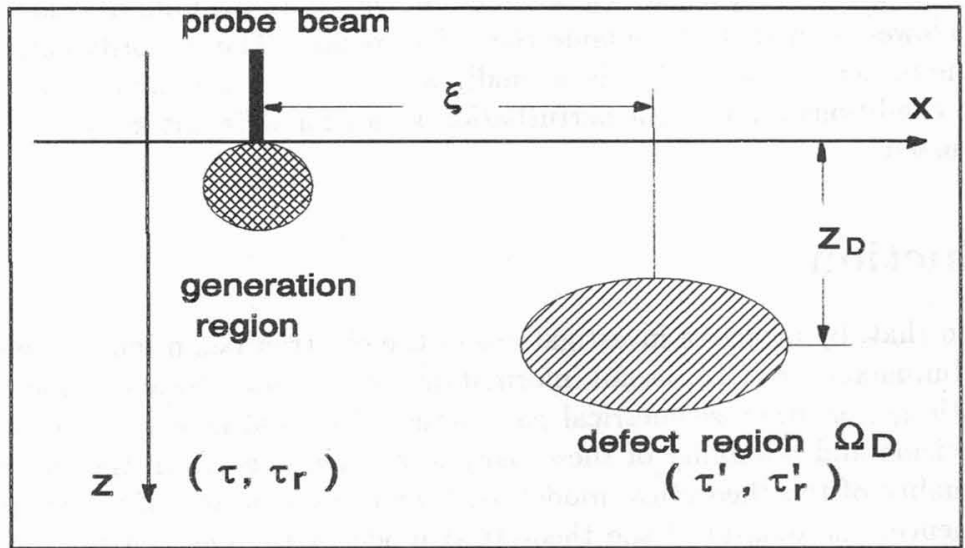

Figure 1: Schematic view of an experiment for the measurement of defect contrasts

If the probe beam is positioned near the defect region at the point $\xi$ one measures a luminescence signal $I^{L}(\xi)$ or an induced current $I^{c}(\xi)$ which is different from the corresponding signal $I_{0}$ in the defect-free region, i.a. far away from the dislocation. The contrast can be determined by

$$
c(\xi)=\frac{I(\xi)-I_{0}}{I_{0}}
$$

For the calculation of the signal near the defect it is necessary to know the concentration of the minority carriers near the defect. If low injection conditions are assumed, the minority carrier density obeys the following continuity equation

$$
D \nabla^{2} q(\vec{r})-\frac{1}{\tau} q(\vec{r})=-g(\vec{r})+\left[\frac{1}{\tau^{\prime}}-\frac{1}{\tau}\right] \theta(\vec{r}) q(\vec{r})
$$

$\theta(\vec{r})$ is given by

$$
\theta(\vec{r})=\left\{\begin{array}{lll}
1 & : & \vec{r} \in \Omega_{D} \\
0 & : & \vec{r} \notin \Omega_{D}
\end{array} .\right.
$$


$\Omega_{D}$ denotes the volume of the defect. $q(\vec{r})$ has to fulfil boundary conditions corresponding to the geometry of the problem. $q(\vec{r})$ has to vanish far away from the generation region and far away from surfaces. At surfaces and interfaces the boundary conditions have the usual form

$$
D \frac{\partial q(\vec{r})}{\partial n}=v_{s} q(\vec{r})
$$

( D - diffusion coefficient, $v_{s}$ - surface recombination velocity)

If the Green function $G\left(\vec{r}, \vec{r}^{\prime}\right)$ of equation (2) is known, the solution of (2) can be written in form of an integral equation

$$
q(\vec{r})=q_{0}(\vec{r})+\gamma \int \Omega_{D} d^{3} \vec{r}^{\prime} q\left(\vec{r}^{\prime}\right) \frac{G\left(\vec{r}, \vec{r}^{\prime}\right)}{4 \pi L^{2}} \quad, \quad \gamma=\left[1-\frac{\tau}{\tau^{\prime}}\right] .
$$

The most common way to solve this integral equation is to write down $q(\vec{r})$ in form of a perturbation series

$$
\begin{aligned}
q(\vec{r})= & q_{0}(\vec{r})+\gamma \int \Omega_{D} d^{3} \vec{r}_{1} q_{0}\left(\vec{r}_{1}\right) \frac{G\left(\vec{r}, \vec{r}_{1}\right)}{4 \pi L^{2}}+\sum_{n=2}^{\infty} \gamma^{n} \int_{\Omega_{D}}^{(1)} \ldots \int_{\Omega_{D}}^{(2)} q_{0}\left(\vec{r}_{n}\right) \times \\
& \times \frac{G\left(\vec{r}, \vec{r}_{1}\right)}{4 \pi L^{2}} \prod_{j=1}^{n-1}\left[\frac{G\left(\vec{r}_{j}, \vec{r}_{j+1}\right)}{4 \pi L^{2}}\right] d^{3} \vec{r}_{n} .
\end{aligned}
$$

\section{The properties of the perturbation series}

Equation (4) is a Fredholm integral equation of second kind for the minority carrier density $q(\vec{r})$ in the presence of a defect. The kernel of this integral equation $K\left(\vec{r}, \vec{r}^{\prime}\right)$ is given by the Green function

$$
K\left(\vec{r}, \vec{r}^{\prime}\right)=G\left(\vec{r}, \vec{r}^{\prime}\right) /\left(4 \pi L^{2}\right)
$$

The kernels corresponding to the problems of contrast calculations are weak polar ones. From the theory of integral equations [10] it follows that if $|\gamma|<1 / N$ (with $\gamma$ from (4)) the solution can be written as a Neumann series, which is uniform convergent. The quantity $N$ is given by

$$
N=\max _{\vec{r} \in \Omega_{D}} \int \Omega_{D} \frac{G\left(\vec{r}, \vec{r}^{\prime}\right)}{4 \pi L^{2}} d^{3} \vec{r}^{\prime}
$$

Therfore we can conclude

- not for every value of $\gamma$, that means not for all values of the defect strength it is possible to solve the problem with the help of a Neumann series,

- if it is not possible to use a Neumann series, there is no certainty that a first order consideration will provide a correct result,

- only the change of the total minority carrier lifetime enters this discussion of convergence. There are no limitations for the inclusion of changes of the radiative lifetime or of the absorption coefficient near the defect ( $\mathrm{cf} .[1,6,8])$.

Another result of the theory of integral equations is the existance of a majorant series. This majorant series can be used to get information on the speed of convergence on the one hand, 
and on the deviation of the first order approximation from the exact result on the other. If a perturbation series exists, we can estimate the error in the first order approximation by

$$
q^{\text {exact }}(\vec{r})-q^{1 . o r d e r}(\vec{r}) \leq \max _{\vec{r} \in \Omega_{D}} q_{0}(\vec{r}) \frac{1}{1-\gamma N}
$$

The error decreases and the speed of convergence increases if the product $\gamma N$ decreases.

For some instrucutive cases we want to evaluate the criterion of convergence.

\subsection{Defects in bulk material}

At first, defects far away from any boundaries are considered. Therefore only quantities, which describe the defect enter the criterion of convergence. This situation does not correspond directly to an experimental situation, but we can get the results easily.

If we consider a sperical defect, the quantity $\mathrm{N}$ depends only on the volume of the defect region.

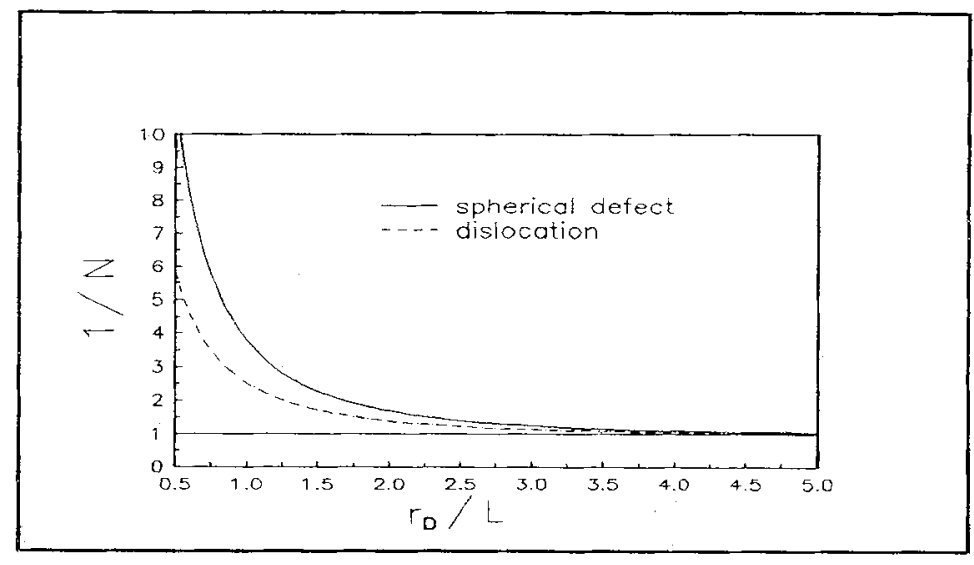

Figure 2: Criterion of convergence for defects in bulk material ( spherical defect, dislocation )

From reasons of symmetry it follows that $\mathrm{N}$ will reach the maximum value if $\vec{r}$ corresponds to the center of the sphere. So, we get from (6)

$$
\left|\left(\frac{\tau}{\tau^{\prime}}-1\right)\right|<\frac{1}{N}=\left[1-\left(\frac{r_{D}}{L}+1\right) \exp \left(-\frac{r_{D}}{L}\right)\right]^{-1} .
$$

Using some results of analytical calculations of defect contrasts [11] it is also possible to evaluate the criterion of convergence for a dislocation in the infinite space. The dislocation is simulated by a cylindrical defect region. The radius of the defect is the geometrical quantity which enters the criterion. We get

$$
\left|\left(\frac{\tau}{\tau^{\prime}}-1\right)\right|<\frac{1}{N}=\left[1-\frac{r_{D}}{L} \mathrm{~K}_{1}\left(\frac{r_{D}}{L}\right)\right]^{-1} .
$$

( $K_{\nu}$ - modified Bessel function of second kind of order $\nu$ )

The quantity $1 / \mathrm{N}$ in dependence on the defect radius for these two defect configurations is shown in Fig. 2 . The allowed deviation of the minority carrier lifetime from the matrix value decreases if the ratio of the radius of the defect to the diffusion length $\left(r_{D} / L\right)$ increases . 


\subsection{Defects near surfaces}

More interesting for the interpretation of real experiments are configurations like that shown in Fig. 1 schematically. Defect configurations which can be considered in an easy way are, a sperical defect ( radius $-r_{D}$ ) in a distance of $z_{D}$ from the surface, a dislocation ( radius $-r_{D}$ ) perpendicular to the surface, and a dislocation ( radius $-r_{D}$ ) in a distance of $z_{D}$ parallel to the surface.

We restrict our considerations to the case of very small surface recombination velocity ( $v_{s}=$ $\left.0, \epsilon_{s}=1\right)$ and an infinite surface recombination velocity $\left(v_{s} \rightarrow \infty, \epsilon_{s}=-1\right)$.

In contrast to the defects in the bulk, where the maximum of $\mathrm{N}$ follows from reasons of symmetry, the position of $\vec{r}$ (cf. (6) ) within the defect region is dependent on surface recombination velocity and distance from the boundary. So one has to evaluate (6) numerically.

We want to consider dislocations parallel to the surface. For this case we can evaluate (6) analytically. From reasons of symmetry it follows that $\vec{r}$ lies on the z-axis. We get

$$
\left|\left(\frac{\tau}{\tau^{\prime}}-1\right)\right|<\frac{1}{N}=\left\{\max _{\vec{r} \in \Omega_{D}}\left[1-\frac{r_{D}}{L} \mathrm{I}_{0}\left(\frac{\left|z-z_{D}\right|}{L}\right) \mathrm{K}_{1}\left(\frac{r_{D}}{L}\right)+\epsilon_{s} \mathrm{~K}_{0}\left(\frac{z+z_{D}}{L}\right) \mathrm{I}_{1}\left(\frac{r_{D}}{L}\right) \frac{r_{D}}{L}\right]\right\}^{-1} .
$$

$\left(I_{\nu}-\right.$ modified Bessel function of first kind of order $\nu$ )

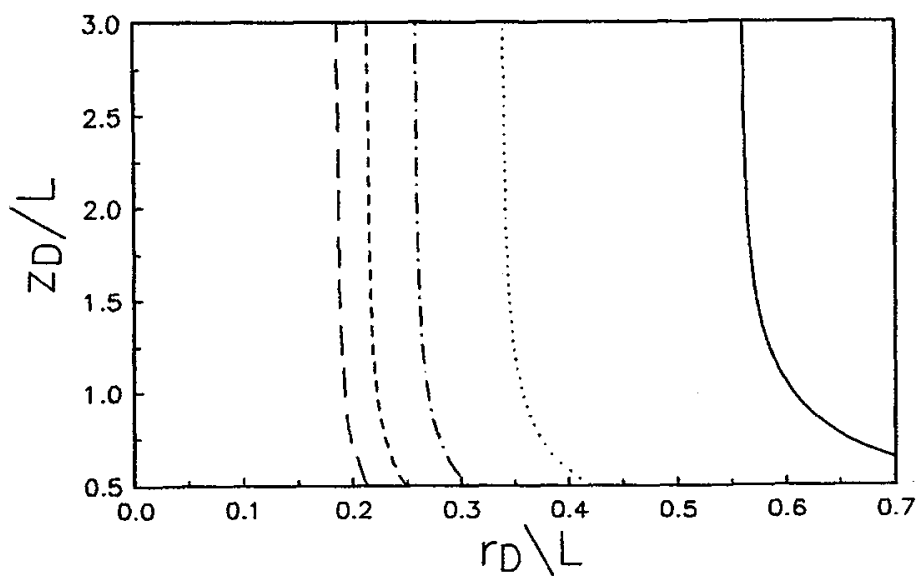

Figure 3: Criterion of convergence for a dislocation located parallel to a surface. $\epsilon_{s}=-1$ was used. A contour plot for $N^{-1}$ is given ( solid line $N^{-1}=25$, dotted line $N^{-1}=20$, dashed dotted line $N^{-1}=15$, short dashed line $N^{-1}=10$ and long dashed line $N^{-1}=5$ )

Numerical tests have shown, that it is a good approximation to assume $z=z_{D}$, independent of the surface recombination velocity and the depth position of the dislocation. Then (10) can be further simplified. We get

$$
\left|\left(\frac{\tau}{\tau^{\prime}}-1\right)\right|<\frac{1}{N}=\left[1-\frac{r_{D}}{L}\left\{\mathrm{~K}_{1}\left(\frac{r_{D}}{L}\right)-\epsilon_{s} \mathrm{~K}_{0}\left(\frac{2 z_{D}}{L}\right) \mathrm{I}_{1}\left(\frac{r_{D}}{L}\right)\right\}\right]^{-1} .
$$

Fig. 3 shows a contour plot corresponding to $N^{-1}$. In this case the surface recombination velocity is large $\left(v_{s} \tau / L \rightarrow \infty\right)$. For a certain $N^{-1}$ the perturbation series is convergent, if the geometrical quantities of the dislocation define such a point in the graph, that the point lies on the left-hand side of the contour of the corresponding value of $N^{-1}$. 


\section{Concluding Remarks}

It was illustrated that the use of a perturbation series in the contrast calculations is restricted to a domain of convergence, which is dependend on the geometrical quantities of the experimental situation and the change of the minority carrier lifetime in the defect region with respect to the defect-free sample. So one has to be carfully in using such approximations for an interpretation of experimental results and for the definition of quantities like the defect strength.

\section{References}

[1] HILDEBRANDT,S. and HERGERT,W., phys. stat. sol. (a) 119(1990)689

[2] DONOLATO,C., Optik 52(1978)19

[3] DONOLATO,C., phys. stat. sol. (a) 65(1981)649

[4] DONOLATO,C., Journal de Physique 44-C4(1983) 269

[5] DONOLATO,C. and BIANCONI,M., phys. stat. sol. (a) 102(1987)K7

[6] PASEMANN,L. and HERGERT,W., Ultramicroscopy 19(1986)15

[7] PASEMANN,L., Ultramicroscopy 6(1981)237

[8] PASEMANN,L., Thesis B, Leipzig 1987

[9] WEBER,G., DIETRICH,S. HÜHNER,M., ALEXANDER, H., Inst.Phys.Conf.Ser. 100 (1989) 749

[10] WLADIMIROW,W.S., Gleichungen der mathematischen Physik, Berlin 1972

[11] HERGERT,W., Thesis B, Halle 1988 